\title{
latrogenic giant left anterior descending coronary artery pseudoaneurysm with contained perforation following percutaneous coronary intervention
}

Giant iatrogenic coronary pseudoaneurysms are very rare. Left untreated, they may cause serious adverse outcome. We present a case of a male patient with recurrent NSTEMIs and coronary interventions who developed a large LAD artery pseudoaneurysm with contained rupture. He underwent excision of the large proximal LAD pseudoaneurysm, excision of the previous LAD stent, ligation of proximal LAD, and coronary artery bypass grafting with LIMA-LAD graft. He subsequently recovered and was stable on follow up. In conclusion, an aggressive surgical strategy, though difficult, is likely the best means of management for lasting benefit.

Keywords: Giant pseudoaneurysm - Coronary intervention - Left anterior descending

\section{Abbreviations}

PCI: Percutaneous Coronary Intervention; NSTEMI: Non-ST-elevation Myocardial Infarction; LAD: Left Anterior Descending; TTE: Transthoracic Echocardiography; LV: Left Ventricle; OM: Obtuse Marginal; CABG: Coronary Artery Bypass Grafting; LIMA: Left Internal Mammary Artery; CPB: Cardiopulmonary Bypass; AXC: Aortic CrossClamp; IABP: Intra-Aortic Balloon Pump; TEE: Transoesophageal Echocardiography; IVUS: Intravascular Ultrasound; RV: Right Ventricle

\section{Introduction}

Chronic iatrogenic giant coronary artery pseudoaneurysms are very rare. They generally occur after repeated and often complex percutaneous coronary intervention (PCI) but may occur following pericardiocentesis [1]. Spontaneous giant coronary artery pseudoaneurysms have been reported following inflammatory disease [2,3] and blunt trauma [4]. However, scarce literature exists regarding giant coronary artery pseudoaneurysms complicated by contained rupture and their management.

\section{Case Report}

A 62-year-old Bangladeshi male smoker with past medical history of hypertension, type II diabetes mellitus suffered non-ST elevation myocardial infarctions (NSTEMIs) in Apr and Oct 2017 for which he underwent drug-eluting stent (DES) deployment to the proximal left anterior descending artery (LAD) and successful angioplasty of an occluded LAD from late stent thrombosis followed by deployment of a second stent respectively. Coronary angiogram then showed a tiny outpouching at the proximal LAD which was managed conservatively. In May 2018, he developed another NSTEMI and presented to our institution for second opinion in Jun 2018. His medications included a statin, aspirin, prasugrel and betablocker.
Mofassel Uddin Ahmed', Jai Ajitchandra Sule ${ }^{1 *}$, Kang Giap Swee ${ }^{1}$, Li Quan² and Theodoros Kofidis $^{1}$

'Department of Cardiac, Thoracic and Vascular Surgery, Division of Cardiac Surgery, National University Heart Centre Singapore, National University Hospital, Singapore

2Department of Cardiology, National University Heart Centre Singapore, National University Hospital, Singapore

*Author for correspondence: E-mail: roversage@gmail.com Received date: February 27, 2019 Accepted date: March 25, 2019 Publication date: April 02, 2019 
He was mildly cachectic but symptom-free. Transthoracic echocardiography (TTE) revealed left ventricle (LV) ejection fraction of $45-50 \%$ with no valvular lesion. Coronary angiogram showed occluded proximal left anterior descending (LAD) coronary artery including the previously stented segment, a discrete $75 \%$ ostial first obtuse marginal (OM) lesion, and extravasation of contrast into a large pseudoaneurysm. This presented itself as a walled up pericardial mass on echocardiography (Figure 1). He subsequently underwent surgical repair via sternotomy.

Upon pericardiotomy intraoperatively, we identified an area of dense adhesions to the left lateral pericardium, which upon dissection, was a contained LV wall rupture, with local thrombosis. After opening the large $7 \times 5.5 \mathrm{~cm}$ pseudoaneurysm cavity, we found the latter extended deep into the septum but fortunately did not break through into the LV or RV. The LAD was occluded proximally and the stent was completely thrombosed (Figure 2).

We performed excision of the large proximal LAD pseudoaneurysm, excision of LAD stent, ligation of proximal LAD, and coronary artery bypass grafting (CABG) of the distal-mid LAD with left internal mammary artery (LIMA) graft (CPB 138 minutes, AXC 73 minutes). Intra-aortic balloon pump (IABP) was inserted empirically prior to weaning $\mathrm{CPB}$ due to large areas of LV anterior wall scar tissue and occluded LAD.

The distal LAD $(1.5 \mathrm{~mm})$ was patent but the OM1 was too small to graft. Post-procedure transoesophageal echocardiography (TEE) showed ejection fraction of $40 \%$.
There was extensive ooze from raw adhesion surfaces and vascularized epicardial tissue. Hence, we packed the pericardial space temporarily with hemostatic agents and performed sternal closure. The patient developed impending cardiac tamponade 5 hours later and was brought back to operation theatre for resternotomy. About $300 \mathrm{ml}$ of clotted blood was found on the posterolateral surface of the heart and no active bleed. MiraQ ${ }^{\mathrm{TM}}$ Cardiac (Medistim ASA, Plymouth, Minnesota, USA) transit time flow measurement showed satisfactory flow values and pulsatility index of the LIMA-LAD graft.

Postoperatively, he developed transient delirium and long QT syndrome which subsequently resolved. His recovery was otherwise uneventful and he discharged well on the $12^{\text {th }}$ postoperative day. Histology was consistent with pseudoaneurysm.

\section{Discussion}

Iatrogenic coronary artery pseudoaneurysms are much less common than coronary aneurysms and usually occur after catheter-based coronary interventions as a result of traumatic dissection or perforation of a coronary artery [5-7]. They are also a rare complication following the implantation of a DES [8-10], with a reported association with stent fracture [11-13]. They are often detected on repeat angiography for symptoms of ischemia or myocardial infarction [11]. Intravascular ultrasound (IVUS) may be used to differentiate pseudoaneurysms from aneurysms $[14,15]$ and identify those at higher risk for complications [10].

While the precise incidence of iatrogenic pseudoaneurysms as well as the exact pathophysiology causing them remains unknown, many are believed to

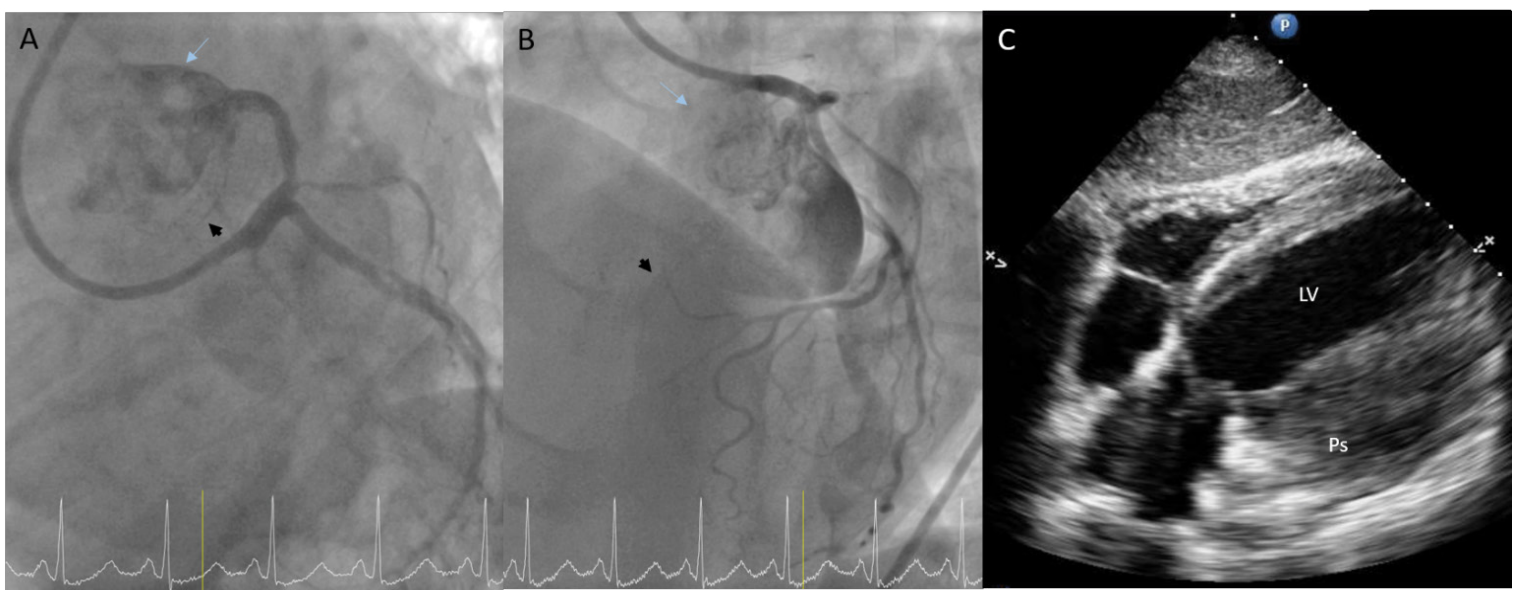

Figure 1: (A) LAO caudal and (B) cranial views demonstrating contrast extravasation into a large LAD pseudoaneurysm (light blue arrows). Outline of the proximal LAD stent (black arrows) is visible with no flow down the LAD. (C) Subcostal view showing the pseudoaneurysm appearing as a walled up pericardial mass. LV: left ventricle. Ps: pseudoaneurysm. 


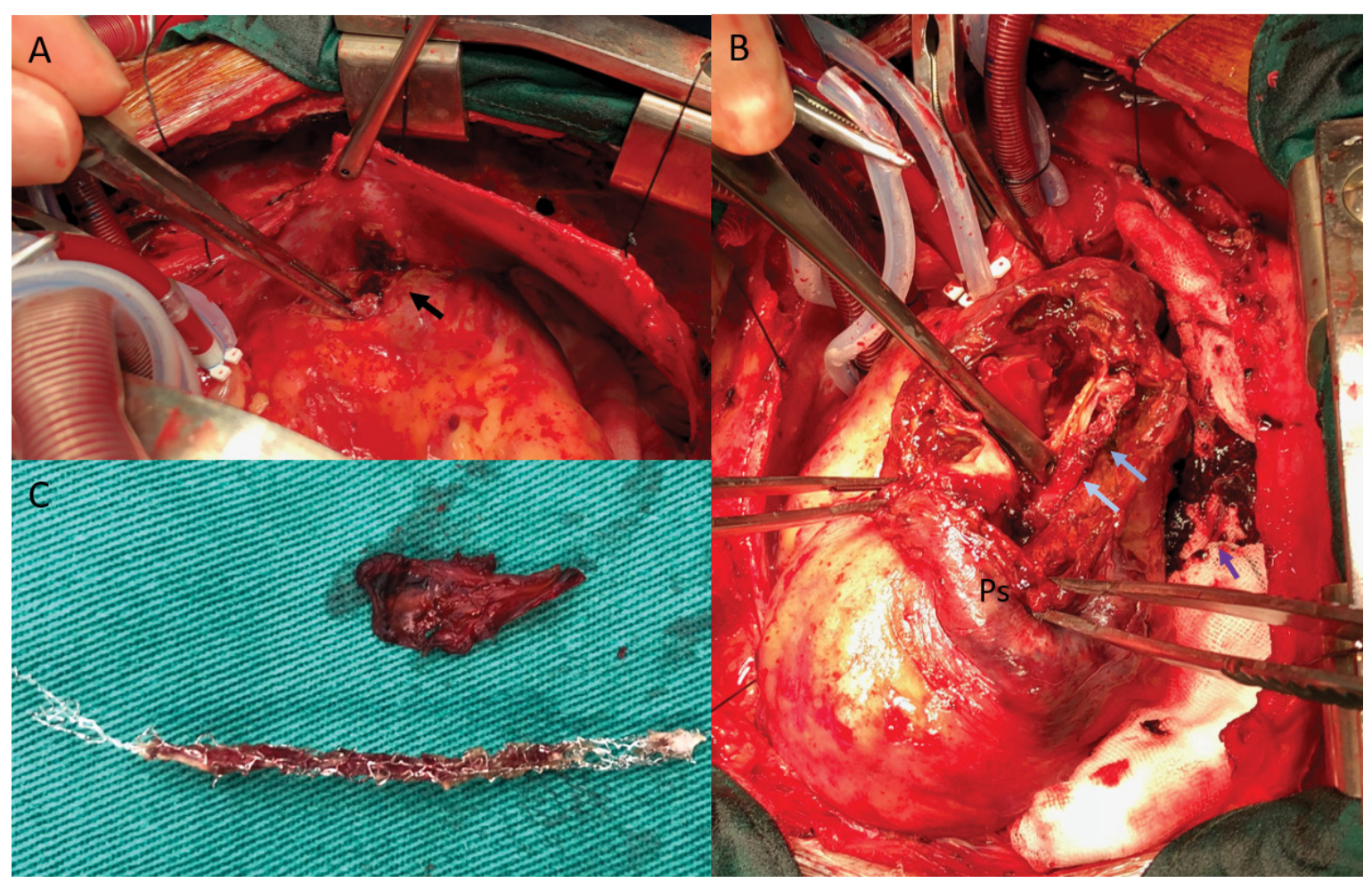

Figure 2: (A) Large pseudoaneurysm of the proximal LAD eroding through the LV wall (black arrow) with dense adhesions to the pericardium. (B) Totally thrombosed and exposed (extravascular) stent (blue arrows) dissected from within the proximal LAD and freefloating within the pseudoaneurysm sac. Extensive clots seen to erode into the LV epicardium (purple arrow). Ps: pseudoaneurysm. (C) Excised stent and thrombosed portion of proximal LAD pseudoaneurysm.

be caused by traumatic injury to the arterial wall $[16,17]$ or infection $[18,19]$. Additional complex inflammatory or hypersensitivity triggers and incomplete endothelialisation may be possible etiologies in the case of pseudoaneurysms after DES implantation [15].

Pseudoaneurysms are subject to various phasic strain and stress forces that contribute to their development. Recently, the size of an aortic subvalvular pseudoaneurysm has been shown to vary with the cardiac phase on synchronized CT angiogram [20]. Studying the structural effects of stress on coronary pseudoaneurysms in relation to such imaging may aid in determining the timing and choice of therapy. c-Jun N-terminal kinase (JNK) is a stress-response kinase and its activation in response to various cellular stresses is observed in various cardiac diseases $[21,22]$. Interleukin-34 (IL-34) is a pro-inflammatory factor closely associated with coronary artery diseases and atherosclerosis [23]. These novel factors can be potential targets for research in the natural progression of coronary pseudoaneurysms at the proteomic level.

Management strategy depends on the size of the pseudoaneurysm, presence of any secondary complication as well as the patency of the native coronary artery or existing stent. Smaller pseudoaneurysms may close spontaneously with observation alone $[6,24,25]$. They may be treated via PCI using PTFE [26-28] or pericardial [29] covered stents or with percutaneous coil embolization [30], and surgical strategy includes on-lay patch grafting which can be done off-pump [31].

Large pseudoaneurysms are more likely to pose a risk of serious adverse outcomes including rupture and cardiac tamponade, coronary artery thrombosis and stenosis, distal embolization, fistulation into adjacent structures [32] and infection if left untreated and warrant early intervention. Treatment for a leaking pseudoaneurysm includes PCI using covered stents versus surgical repair with cardiopulmonary bypass or extracorporeal membrane oxygenator support. Contained rupture of large pseudoaneurysms may also create lobulated pseudoaneurysms that may be prone to future rupture [33]. Surgery is aimed at coronary artery ligation at both ends of the pseudoaneurysm, resection of the pseudoaneurysm and distal CABG [34].

In this case, extensive adhesions and erosion through the epicardium made identifying the LAD difficult. The LAD course was identified by passing a probe retrograde via a distal LAD arteriotomy. Scar ends of epicardium around the pseudoaneurysm were closed primarily in 2 layers, buttressed with $\mathrm{TachoSil}^{\bullet}$ (Baxter, Deerfield, 
Illinois, USA). Two favourable features were first, the LV wall rupture was contained by pericardial adhesions and second, the pseudoaneurysm was contained within thick septal wall rather than fistulate into the LV or RV; the latter would have seriously aggravated the course and potentially outcome of his surgery.

\section{References}

1. Yerramareddy VC. A rare case of iatrogenic coronary pseudoaneurysm following pericardiocentesis. IHJ Cardiovascular Case Reports. 1(2): 83-85 (2017).

2. $\mathrm{Pu} \mathrm{L}$. A giant pseudoaneurysm of coronary artery in a young patient with Behçet's disease. Echocardiography. 34(11): 17361737 (2017).

3. Harrison A. Cardiovascular complications in behçet syndrome acute myocardial infarction with late stent thrombosis and coronary, ventricular, and femoral pseudoaneurysms. Tex Heart Inst J. 36(5): 498-500 (2019).

4. Liu L. Coronary artery pseudoaneurysm following blunt trauma. J Cardiac Surg. 27(5): 563-565 (2012).

5. Flum DR, McGinn JT, Tyras DH, et al. Coronary pseudoaneurysm after angioplasty. Am Surg. 61: 1035-1038 (1995).

6. Kawazoe H. Spontaneous disappearance of coronary pseudoaneurysm due to coronary artery perforation following percutaneous coronary intervention. Cardiovas intervand therapeut. 28: 408-414 (2013).

7. Schöbel WA. Occurrence of a saccular pseudoaneurysm formation two weeks after perforation of the left anterior descending coronary artery during balloon angioplasty in acute myocardial infarction. Catheter Cardiovasc Interv. 47(3): 341346 (1999).

8. Ichikawa M, Kijima Y. Spontaneous resolution of pseudoaneurysm after zotarolimus-eluting stent implantation: imaging evidence at 13 months of follow-up. Catheter Cardiovasc Interv. 30(2): 168-170.

9. Chen D. Spontaneous resolution of coronary artery pseudoaneurysm consequent to percutaneous intervention with paclitaxel-eluting stent. Tex Heart Inst J. 35(2): 189-192 (2008).

10. Alfonso F. Coronary aneurysms after drug-eluting stent implantation: clinical, angiographic, and intravascular ultrasound findings. JACC 53(22): 2053-2060 (2009).

11. Kawai Y. A case of coronary rupture and pseudoaneurysm formation after fracture of implanted paclitaxel-eluting stents. Cardiovas Interv TherApeut. 31(3): 231-237 (2016).

12. Sandhu PS, Kaul U. Coronary stent fracture resulting in pseudoaneurysm. Indian Heart J. 64: 622-623.

13. Choi JH, Song BG, Song YB, et al. Catastrophic coronary stent fracture and coronary perforation presenting as cardiogenic shock: a rare but fatal complication of stenting. Circ Cardiovasc Imaging. 1: 7-8 (2008).

14. Aqel RA. Spontaneous coronary artery dissection, aneurysms, and pseudoaneurysms: review. Echocardiography. 21(2): 175-82 (2004).

\section{Conclusion}

An aggressive and timely surgical strategy is likely the best means of managing large complex coronary pseudoaneurysms for lasting benefit.

15. Aoki J. Coronary artery aneurysms after drug-eluting stent implantation. JACC: Cardiovasc Interven. 1(1): 14-21 (2008).

16. Bell MR. Relation of deep arterial resection and coronary artery aneurysms after directional coronary atherectomy. J Am Coll Cardiol 20: 1474-1481 (1992).

17. Dralle JG. Coronary artery aneurysms after angioplasty and atherectomy. Ann Thorac Surg. 59: 1030-1035 (1995).

18. Le MQ, Narins CR. Mycotic pseudoaneurysm of the left circumflex coronary artery: a fatal complication following drugeluting stent implantation. Catheter Cardiovasc Interv. 69: 508512 (2007).

19. Furtado AD, Bhat SPS, Peer SM, et al. Infected pseudoaneurysm involving a drug-eluting stent. Interact CardioVasc Thorac Surg. 12: 636-638 (2011).

20. Svensson A. Variable size of aortic subvalvular pseudoaneurysm. Acta Radiol Open. 7(6): 205-284 (2018).

21. Yan J. Stress signaling JNK2 crosstalk with CaMKII underlies enhanced atrial arrhythmogenesis. Circ Res. p: 16 (2019).

22. Yan J. Role of stress kinase JNK in binge alcohol-evoked atrial arrhythmia. J Am Coll Cardiol. 71(13): 1459-1470 (2018).

23. Qingyan Liu. IL-34 promotes foam cell formation by enhancing CD36 expression through p38 MAPK pathway. Sci Rep. 8: 17347 (2018).

24. Nunes RA. Spontaneous closure of post-intervention left anterior descending coronary pseudoaneurysm. Rev Port Cardiol. 33(6): 381 (2014).

25. Mikhail B, Brewer RJ, Clark VL, et al. Spontaneous closure of a perforation-induced coronary artery pseudoaneurysm. J Invas Cardiol. 14: 282-284 (2002).

26. Lell E. Delayed development of a coronary artery pseudoaneurysm after angioplasty. Cathet ardiovasc Intervent. 47: 186-190 (1999).

27. Amano T, Nakamura S. A Case of percutaneous coronary intervention using a polytetrafluorethylene-covered stent for an iatrogenic pseudoaneurysm of the left main coronary artery. $J$ of Cardiol and Vasc Med. 3: 1-5 (2018).

28. Johnson NP. Post-intervention coronary pseudoaneurysm treated with a covered stent. Tex Heart Inst J. 39(3): 448-449 (2012).

29. Bogaard K. Coronary artery pseudoaneurysm: closure with pericardium-covered stents, guided by cardiac computed tomography angiography. Canadian J of Cardiol. 29: 1014-1014 (2013).

30. Sallam T. Coil embolization of left coronary artery pseudoaneurysms arising as a complication of percutaneous coronary intervention. Catheter Cardiovasc Interv. 80(7): 12281231 (2012). 
31. Sasaguri S. A surgical case report of off-pump onlay patch grafting for pseudoaneurysm with diffusely calcified coronary artery. Ann Thorac Cardiovasc Surg. 17: 94-96 (2011).

32. Hagau AMT. Large coronary pseudoaneurysm with pulmonary artery fistula, six months after left main trunk stenting with paclitaxel-eluting stent. Med Ultrason. 15(1): 59-62 (2013).
33. Cabarrus MBS. Iatrogenic giant coronary artery pseudoaneurysm with "daughter aneurysm" formation: serial imaging findings and natural history. J Thorac Imaging. 27(6): 185-7 (2012).

34. Aggarwal P. Successful management of a giant unruptured mycotic coronary artery aneurysm after coronary angioplasty. Indian Heart J. 68(2): 44-46 (2016). 